
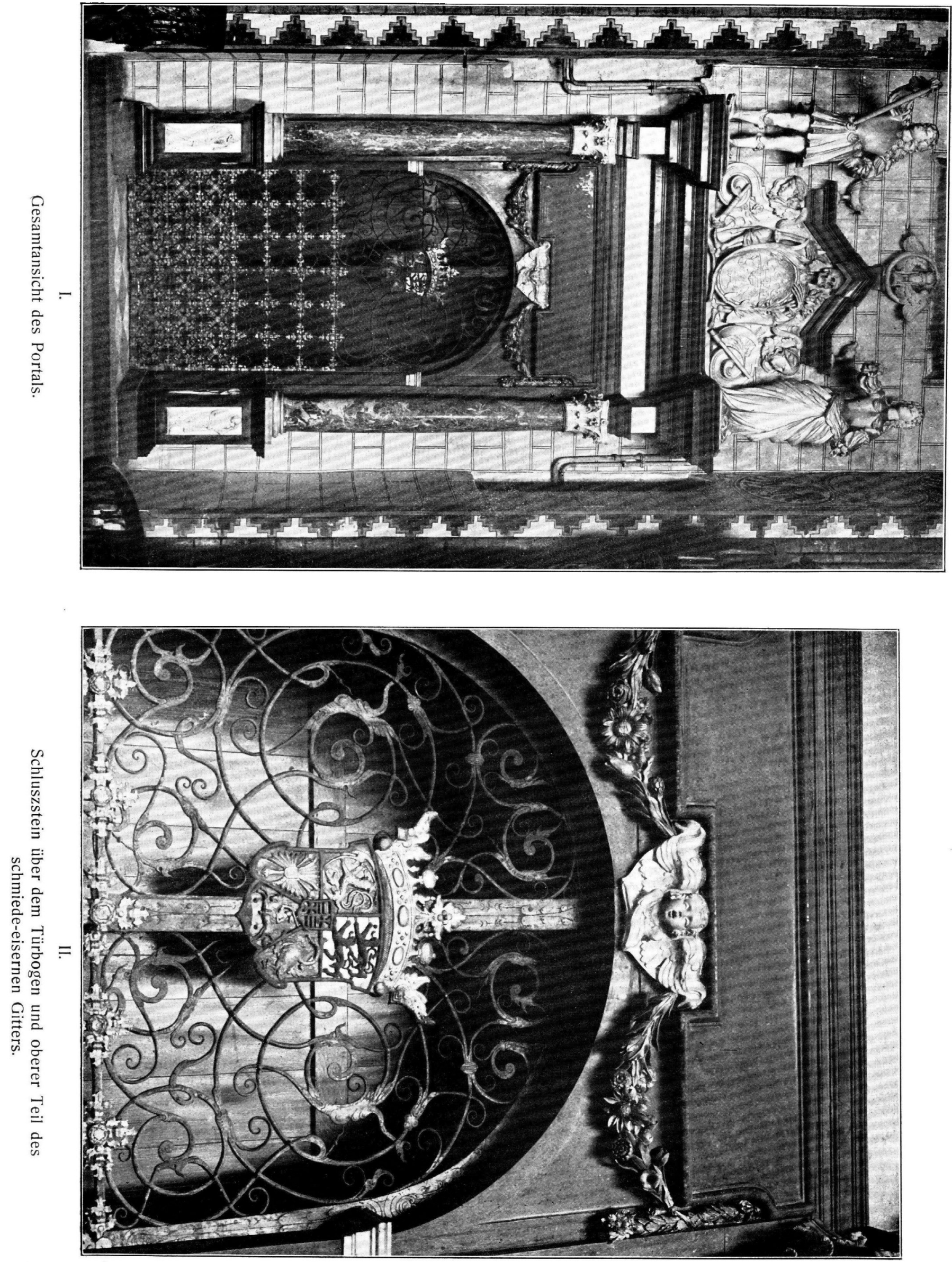


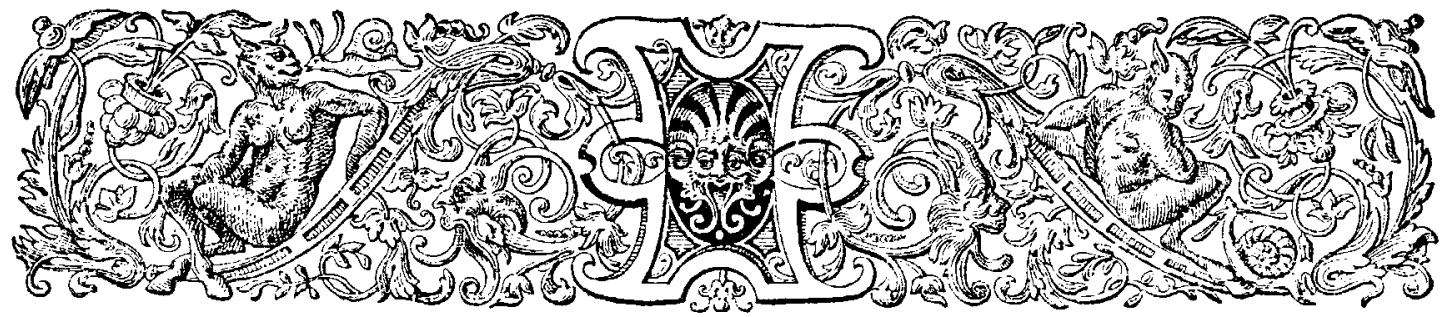

\title{
DAS PORTAL DER HERZOGLICHEN GRUFT IM DOM ZU SCHLESWIG, EIN WERK DES ARTUS QUELLINUS. VON
}

\author{
DR. HARRY SCHMIDT.
}

(Flensburg.)

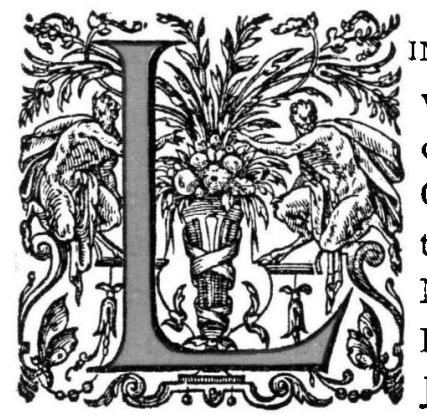

INks von dem herrlichen Altarwerke HANs BRüGGEMANNS von 152 I erhebt sich an der Nordostseite des Chorraumes des Domes zu Schleswig das Portal der herzoglichen Gruft. Seine Marmorskulpturen können neben dem Meisterwerke des alten Bildschnitzers in Ehren bestehen. Der Name des Künstlers war bisher nicht bekannt. Studien im Reichsarchiv zu Kopenhagen, die vornehmlich dem Maler JükGEN OvENS 1) galten, brachten mir im Sommer I9I2 als unverhoffte Frucht ausser unbekannten Rechnungsbelegen über verschollene Werke Haxs GuDEWERDTs von Eckernförde, eines der bedeutendsten deutschen Bildschnitzer des 17. Jahrhunderts 2), und ausser unbekannten Angaben über Gemälde in Gottorfischen Schlossinventaren des 17. und 18. Jahrhunderts ${ }^{3}$ ) eine Fülle von

1) Ueber Ovens habe ich eine vorläufige, kurz orientierende Skizze mit 9 Bildern im Schleswig-Holsteinischen Kunstkalender Igr 3 veröffentlicht. Das Inventar seines Nachlasses ist im Auszug in Oud-Holland, Igr4, I. Heft veröffentlicht. Eine wortgetreue Wiedergabe des Inventars mit allen Einzelheiten das Originals findet sich in Band 7 der Quellensammlung der Gesellschaft für Schleswig-Holsteinische Geschichte, 1913, S. I-89. Eine abschliessencle Monographie über den auch in seiner Heimat fast vergessenen Meister hoffe ich im nächsten Jahre vorzulegen.

2) u. 3) Diese Archivalia sind im 43. Bande der Zeitschrift der Ges. für Schleswig-Holsteinische Geschichte (IgI3) veröffentlicht.

Oud-Holland, 1914. 
urkundlichen Nachrichten über Künstler des 16-18. Jahrhunderts. Von diesen sind wohl die wichtigsten die Rechnungsbelege der Gottorfischen Rentekam. merbucher, der Ausgabe- und Einnahme-Bücher der Herzöge, uber die Arbeiten des Artus Quellinus. Sie lauten:

I66I. Bezahlung der Gastgeber und Wirthe, Den 6. Junij, No. 448, Hubert Quellin von Ambsterdam, welcher wegen der Fl. Begrebnus im Thumb mit Marmor vnä dergleichen auszuziehren anher erfurdert gezvesen, an aufgewandten Zehrungskosten nach des $\mathrm{Fl}$ : Cantzlern anzeige vnd der quitung No.... entrichtet . . . . . . . . . . . . 25 [Reichstaler].

1662. Extraordinari Auszgaben.

No. 237 den 4. Februarij Johan Danckwerthen, Factorn in Hamburgh erstattet, welche er auf Furstl. Befehlung nacher Ambsterdam an ARTus Qvellin, zegen der Fï̈stl: Erb Begrabniüs in Hiesiger Thumb Kirchen, mit Marmorsteinen inwendig, und auszwendig mit einem Portal dem abrisze nach auszzuzieren einhalt Contracts of 6000 Rthr: Bedung auf abrechnung vbergemachet, ond Bezahlet . . . . . . . . . . . . . 2000 [Reichstaler].

No. 239. den 15 Aprilis und 4 octobr: auff des Herrn Praesidenten anzeige, und Beyverwahrtem 2. Scheine No. 239 Vorgemelden AkTus QUELLIN abermahlen auff abrechnung nacher Ambsterdam übergemachet 2000 [Reichstaler].

1663. Auszgabe an Extraordinari Geldt Poste.

Den 9. Octobris auff des Herrn Praesidenten anmelden, bey vorhandenen Furstl: Contracts und Quitung ARTus Quellin, Steinhowern in Ambsterdam, für die Furstl. Begrebnus in der Thumb Kirchen zu Schleszwigh inwendig mit Marmorsteinen undt zum eingange mit einem Portal auszuzieren und zu verferfertigen über die ihme verschiedentlich vorhin darauff bezahlte und berechnete 4000 Rthr: im entlichen Reste bezahlt. . . . . . 2000 [Reichstaler].

1663. Bezahlung der Gastgeber im Februario 6663.

Den i8. Februarii Johan Martens Gastgeber in Schleszwig was die drey Steinhawer von Amsterdam, welche in hiesiger Fürstl. Begrebnus in der Thumb Kirchen arbeiten sollen, vom i9. Jan. bisz zum 17. Febr. incl. in 29 Tagen bey ihm verzehret, bezahlt . . 33 [Reichstaler] 25 [Schilling] 6 [Pfennig].

I663. Gemeine Auszgaben im Martio den 28. Martij.

JEkE Wilads, Schiffern von Ambsterdam, welcher die Marmorsteine zur 
DAS PORTAL DER HERZOGLICHEN GRUFT IM DOM ZU SCHLESWIG.

Fürstl. begrebnus nach der Friederichstadt geliefert, zur Schiffsfracht vndt Verehrung bezahlt . . . . . . . 52 [Reichstaler] 20 [Schilling].

1663. Gottorffisches Bawwesen.

6. Aprilis $Z$ weyen Taglöhnern welche an der Furstl. Begrebnus im Thumb gearbeitet . . . . . . . . . . . . . . 40 [Schilling].

28. Aprilis. FrancoIS DE SAGER, Steinhowern vom Amsterdam vndt deszen gehülffen an Reise Kosten vnterschietlich, welche ihre Arbeit an der Furstl. Begrebnus in der Thumb Kirchen nunmehr verrichtet laut Quiting bezahlt

65 [Reichsthaler].

Derselben Zehrung vom 18. Febr: bis zum 26. Aprilis bey Ciristian JüTERBOCK Gastgebern in Schleszwig.... entzichtet undt abgehalten mit 50 [Keichstaler] 26 [Schilling].

Io. Decemb: Johan Danckwerth Factorn in Hamburg einhalt Zettels hinwieker erstattet, welche er an JüRGEN OvENs nacher Ambserdam, zegen verlegter asserantz und anderer Vngelder, wegen der daselbst verfertigten Steinhower Arbeit zu Hiesiger Furstl. Erb Begrebnus Vbergemachet hat

I 25 [Reichstaler] 16 [Schillinä].

Aus diesen Angaben der Gottorfischen Rechnungsbücher in Kopenhagen - leider sind die in Schleswig verbliebenen Quittungen und der Kontrakt zwischen dem Fürsten und dem Künstler verloren gegangen - geht hervor: Artus Quellinus I., der Aeltere, der das Rathaus in Amsterdam (den jetzigen Kgl. Palast) mit zahlreichen Marmorskulpturen geziert hat, ist auch der Künstler des furstlichen Erbbegräbnisses im Dom zu Schleswig gewesen. Er hat das Innere des Gruftgewölbes mit Marmor verziert und den Eingang mit einem Portal geschmückt. Diese Arbeiten dauerten von 166I bis 1663. Er erhielt für sie 6000 Reichstaler, nach heutigem Gelde, da der Reichstaler $=3,60 \mathrm{M}$. heutiger Währung ist, 21600 Mark. Der damalige Geldwert ist sicherlich 5 bis 7 mal so hoch anzusetzen wie der heutige, wenn nicht noch höher. Es was also ein wahrhaft fürstliches Honorar. Allerdings zahlten die kunstliebenden Gottorfer Herzöge an hervorragende Künstler, wie zum Beispiel Jürgen OvENs, stets hohe Honorare. QUelitnus hat sich bei der Ausführung an eine Zeichnung, Skizze, gehaiten (dem abrisze nach). Ob diese in Schleswig entstanden ist und er sich an sie anlehnen musste oder ob er nach eigenem Plane arbeitete, ist nicht ersicht. lich. Vermutlich war der sogenannte Abriss sein Vorentwurf, auf Grund dessen er den Auftrag und die Ausführung der Arbeiten erhielt. ARTus ist nicht in Schleswig 
gewesen, wohl aber sein Bruder Hubert Quellinus. $\left.{ }^{1}\right)$ Dieser hat die örtlichen Verhältnisse in Dom durch Augenschein kennen gelernt und die Verhandlungen mit dem Herzog geführt. Er kann nur kurze Zeit dort gewesen sein, denn in den "aufgewandten Zehrungskosten", für die er 25 Reichstaler erhielt, scheintauch die Hin- und Rückreise einbegriffen gewesen zu sein. Daher wäre die Summe fur einen längeren Aufenthalt zu klein. Während dieser Zeit had HUBERT dem Kanzler KielmannseCK gezeichnet, den einflussreichsten Mann am Gottorfer Hofe. Dies Porträt hat RICHARD Colilin später gestochen. Ein Exemplar des, soweit ich sehe, nirgends erwähnten Stiches, durch den wir aüch eine bisher unbekannte Arbeit des HuberT Quellin kennen lernen, sah ich in Schloss Fredriksborg bei Kopenhagen. Das Portal und die innere Ausschmuckung ist im Anfang des Jahres I663 vom I4 Januar bis zum 26 April angebracht von drei Steinmetzen aus Amsterdam, die QuELlinus nach Schleswig sandte. Von diesen war der bedeutendste FRANçOIS DE SA[E]GER. Die Marmorskulpturen, „viele Lasten”, sind zu Schiff von Amsterdam nach Friedrichstadt überführt durch den Schiffer JEkE WILADS aus Amsterdam, wahrscheinlich Ende 1662. Das geht hervor aus einem Empfehlungsschreiben an die Bürgermeister und den Rat der Stadt Friedrichstadt, das der bis 1657 dort wohnhaft gewesene Maler JüRGEN Ovens dem Mons: FR: DE SAEGER mitgab. Dieser bisher unbekannte, in städtischen Archiv in Friedrichstadt befindliche Brief, der einzige Originalbrief des Malers, der später vollständig veröffentlicht werden wird, ist datiert: Amsterdam Den 6. 8bris 1662. Die von mir schon lange gehegte Vermutung, dass Ovens, der für die herzogliche Familie in Amsterdam viele Kunstwerke gekauft hat, auch in diesem Falle dem QUELLINUS gegenüber als eine Art Agent des Herzogs tätig gewesen ist und den Auftrag vermittelt hat, ist durch den letzten urkündlichen Beleg zur Tatsache erhoben. OvEns erhält seine Auslagen [Ongelder] wegen der Marmorskulpturen des QUELLINUS zurück. Ob mit „asserantz” das holländische Wort „assurantie” gemeint ist, sodass die kostbaren Künstwerke durch OveNs versichert worden wären?

Durch Ovens' Vermittlung mögen auch kleinere Arbeiten des QuelLINUS von denen wir bisher keine Kunde hatten, in den Besitz der Gottorfor Herzöge gekommen sein. In einem I7 IO aufgestellten "Inventarium aller in der Hochfürstl: Kunst: und Naturalien Cammer befindlichen Sachen" (Reichsarchiv Kopenhagen) finden sich nämlich folgende Arbeiten des Bildhauers: I. Ein Frauen Zimmer einem Kinde die brust reichende von Thon Modulirt von Quellin, 2. Ein Schild von Kindern gehalten Modulirt von Thon von Quellin, 3. Ein liegendes Kind mit einem Creutz eben der Arbeit und von demselben Meister.

1) Er ist auch sonst für seinen Bruder tätig' gewesen. So veröffentlichte er die Skulptures des Artus im Rathaus zu Amsterdam, die er selbst zeichnete und radierte - in einem Bilderwerk in a Teilen. Der erste erschien r6 $6 \epsilon_{5}$. der zweite 1668 . Diese zahlreichen grossen Blätter zeigen uns HUBERT als tüchtigen Zeichner und Kupferstecher. 


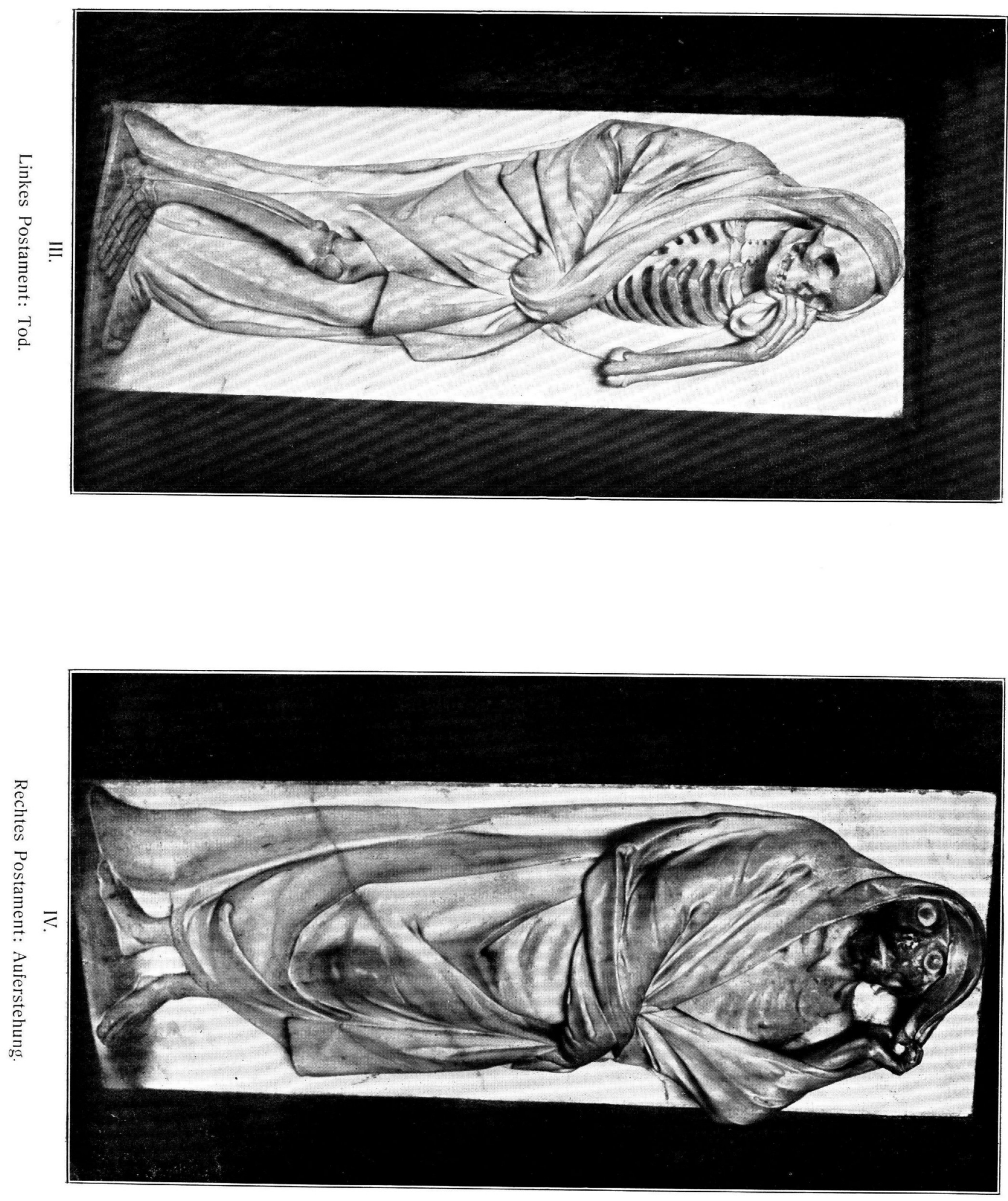
Die Gruft ist von Herzog Christian Albrecht (1659-i694)zum Andenken an seinen Vater, den 1659 in Tönning verstorbenen Herzog FrIEdRICH III. angelegt worden. Die Grabkapelle ist nach HAUPT, (Die Bau- und Kunstdenkmäler der Provinz Schleswig-Holstein, II. S. 293 f.) ein spätgotischer Anbau, dessen Jnneres gegen Ende des 17. Jahrhunderts umgeändert ist. Ursprünglich war sie Sakristei der Geistlichkeit vom Dom. Sie ist von Särgen getüllt, die sich nur durch ihre Grösse auszeichnen, und bietet heute einen Anblick von unerfreulicher Nüchternheit. Beigesetzt sind dort ausser FRIEDRICH III. seine Gemahlin MARIA Elisabeth gest. i684, Christian Albrecht gest, I694 und seine Gemahlin Friderica Amalia, schliesslich FrIEdRICH IV. gest. I702. So war der grosse Raum nur zum Teil gefüllt. Später ist er dann auch als Gruft für die Mitglieder des Holstein-Beckischen (Glücksburger) Hauses benutzt und mit neuen Särgen besetzt.

Die Gruft ist etwa 7,50 im Quadrat, an der Südwestseite ist ein etwa 2 Meter tiefer Vorbau für das Portal. In der Mitte ist das Gewölbe von einer Säule getragen. Die vier gotischen Kreuzgewölbe mit Diagonal- und Gurtrippen von schlichtem Profil endigen in einfachen, verschieden geformten Schluszsteinen in einer Höhe von etwa $6 \mathrm{~m}$ über dem Fussboden. Die Gruft wirt erhellt durch hohes Seitenlicht; je 2 gotische Fenster liegen an der Nord-und Ostseite. Rings herum läuft eine etwa $2 \mathrm{~m}$ hohe Brüstung von grauem Marmor mit weissen Adern, welche die Unterteile der Wände verkleidet. Diese Brüstung wird wohl eine Werkstattarbeit des Quellinus sein, der ja die Gruft ,mit Marmorsteinen inwendig" ausgeziert hat. Besondere künstlerische Vorzüge sind ihr nicht eigen. Die kräftig profilierten Fuss- und Abschluss-Gesimse verkröpfen sich über postamentartigen Platten aus Marmorstuck, von denen je vier an der West-, Nord- und Ost-Seite, zwei an der Süd-Seite angebracht sind. Sie sind etwa I,40 Meter breit und $1,80 \mathrm{~m}$. hoch. Der Vorderseite der Postamente ist je ein ovales Schild aufgelegt. Diese Platten und Schilder stammen vielleicht Z. T. aus der Werkstatt des ARTUS Quellinus, wahrscheinlicher ist es jedoch, dass sie von Thomas Quellinus herrühren. In Nischen darüber sind die Büsten der Bestatteten aufgestelt. An der Nordseite sind es vier Marmorskulpturen, nämlich in der Mitte FrIEDRICH III. und seine Gemahlin, rechts von ihr FrIEDRICH IV. links von ihr Christian Albrecht, neben diesem an der Westseite dessen Gemahlin. Die übrigen Wände, die offenbar zur Aufnahme weiterer Büsten bestimmt waren, sind unbesetzt. HAUPT a. a. O. S. 306 bemerkt: "Zwei der Marmorbüsten sollen von QUELlinus sein," Wahrscheinlich zielt die Ueberlieferung auf THOMAS Quellinus. Wenn damit ARTus gemeint wäre, so widersprechen dem die Rechnungsbücher, die bei ihren genauen Angaben sicherlich etwaige Porträtbüsten 
nicht ausser Acht gelassen hätten. Auch andere Gründe weisen diese Annahme ab. Artus QUellinus, der 1668 starb, könnte doch nur eine Porträtbüste, die des Herzogs FrIEDRICH III. gearbeit haben. Ausserdem stehen die Porträts bei weitem nicht auf der künstlerischen Höhe des Portals. Von Artus QuELLINUs sind sie jedenfalls nicht. Möglicherweise oder wahrscheinlich sind sie von THOMAS, der mit seinem Bruder, dem jüngeren JoHAN ERAsmus Quellinus, Ende des 17. und Anfang des 18. Jahrhunderts lange in Schleswig-Holstein und Dänemark gearbeitet hat und auch für CHRISTIAN AlBRECHT tätig war (S. Haupt a.a.O. S. 356). 1)

Von dem Portal (Gesamtamsicht Abb. I.) das den Eingang zur Gruft bildet, sagt HAUPT, (Domkirche zu Schleswig, S. 21): "Das Portal des herzoglichen Begräbnisses ist im Barockstil gross und stattlich errichtet; die Einzelheizen sind trei ausgeführt und bedeutsam." In dem mehrfach erwähnten Werke heisst es: „Stolzer Eingang im Spätbarock." Den Charakter des Spätsbarocks tragen vornemlich die weiter unten noch $z u$ besprechenden beiden Statuen. Das Portal selbst weist starke Anklange an die holländische Renaissance aut. Das ganze Werk bis zur obersten Spitze ist etwa $61 / 2 \mathrm{~m}$ hoch und etwa $2,80 \mathrm{~m}$ breit. Die Rundbogenöffnung der Tür ist mit schwarzem Marmor bekleidet und flankiert von zwei rötlichen, graugeäderten Marmorsäulen mit weisem korinthischem Kapitäl. Die Säulen werden durch schwarze Marmorpostamente mit weissen Füllungen getragen. Architrav und Oberglieder sind aus schwarzem, der Fries aus weissem Marmor. Das Gebälk verkröpft sich konsolartig über den Säulen. Auf den so gebildeten Vorsprüngen stehen zwei lebensgrosse nach Südwesten blickende Figuren aus Gips, die, wie unten ausgeführt wird, später aufgesetzt sind. Zwischen ihnen hockt der Tod, der das mit Laub umkränzte SchleswigHolsteinische Wappen zwischen seinen Knieen hält. Rechts und links davon zwei schlangenumwundene Frauenköpfe mit Flügeln im Profil. Sie sind von strenger Schönheit. Links eine erlöschende Fackel, rechts eine Sense, die über je eine Schnecke gelegt sind. Rechts wachsen aus der Volute der Schnecke Kornähren, links Blumen hervor, das aus dem Tode emporbluhende Leben symbolisierend. Diese wie andere Symbole erinnern sehr an ähnliche symbolisierende Skulpturen des QueLlinus im Rathause zu Amsterdam. Ueber der Gestalt des Todes erhebt sich eine Spitzverdachung aus schwarzem Marmor, auf der als Seitenbekrönung zwei weisse Schalen stehen. Aus weissem Marmor ist auch die Mittelbekrönung, eine Stundenuhr mit den Flügeln der Zeit, umschlungen von dem Symbole der Ewigkeit, der sich in den Schwanz beissenden Schlange. Be-

1) Wäbrend eines kürzlichen Aufenthalts in Kopenhagen gelang es mir festzustellen, dass diese Statuen samt Platten und Schildern z. T. von einem bisher unbekannten Lübecker Bildhauer HaNS FREESE verfertigt sind. Die urkundlichen Belege hoffe ich im nachsten Jahre vorzulegen. 


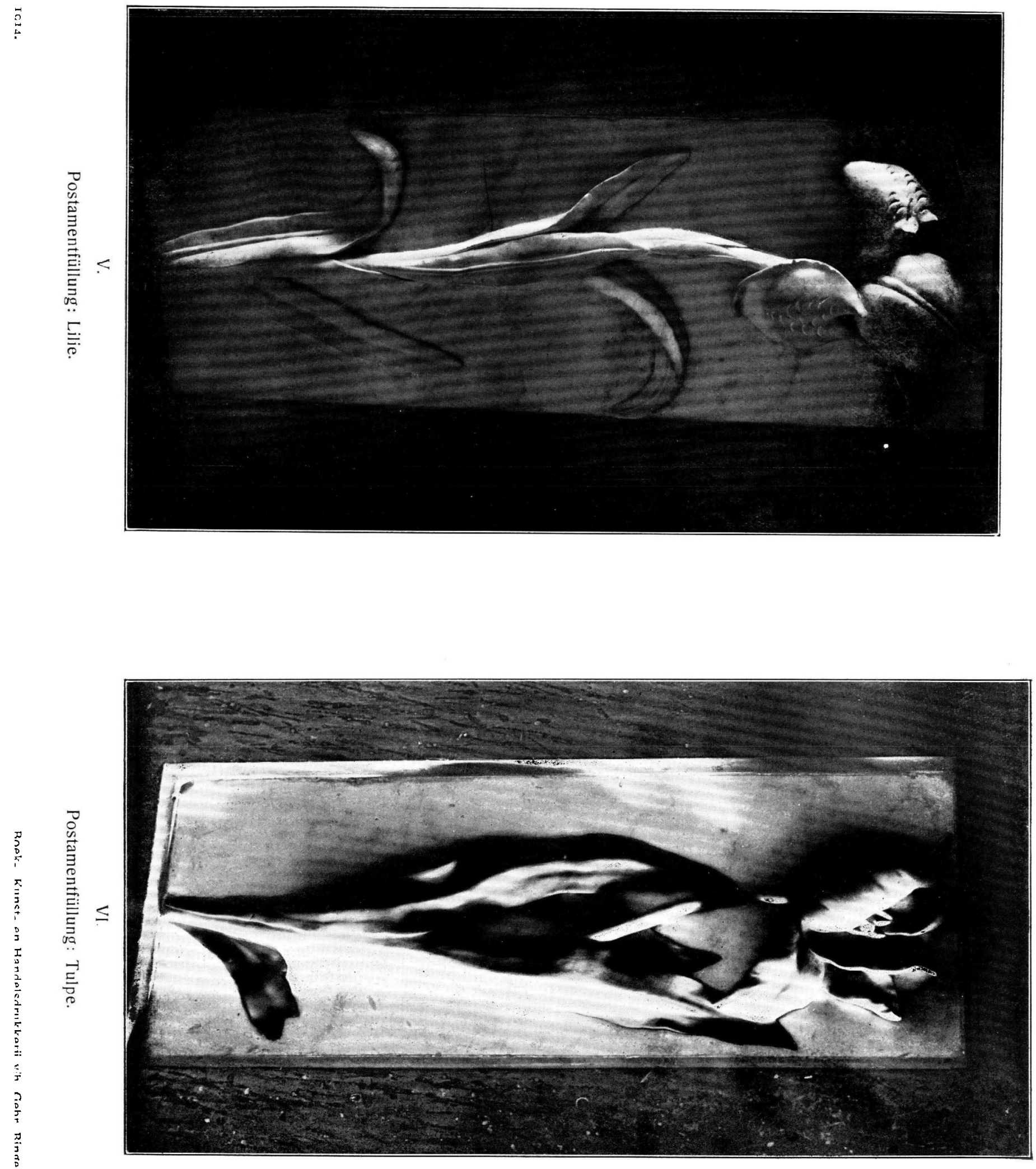
sonders hervorzuheben ist der Schluszstein über dem Türbogen, ein liebliches Engelsköpfchen mit Flügeln, das in die schwarze Füllung reizvoll einschneidet. (Abb.II.) Nach beiden Seiten spannt sich je eine vergoldete Girlande, die seitlich in eine senkrecht herabhängende Girlande abergeht. Die Füllungen der Postamente sind mit je 3 weissen Marmorreliefs geschmückt. die zu den künstlerisch wertvollsten Teilen des bedeutenden Werkes gehören. Auf der Vorderseite des linken Postaments steht der Tod in herkömmlicher Darstellung, im Profil halb nach rechts gewandt (vom Beschauer aus). Er sucht seine Schreckgestalt mit langwallendem Gewande zu verhüllen. (Abb. III.) Auf der Vorderseite des rechts vom Beschauer befindlichen Postaments steht eine andere Gestalt in Vorderansicht, eine sehr originelle Leistung. An sich schon stark realistisch, ist ihr Anblick durch Beschädigungen der Nase und des unteren Teils des Gesichtes geradezu grausig geworden. Eine abgemagerte Gestalt sieht mit grossen tief in den Höhlen liegenden, entsetzt oder erstaunt blickenden Augen uns an. Sie scheint sich der sie ungebenden Hüllen zu entledigen. (Abb. IV.) Zuerst rät man auf Krankheit, Seuche, Pest. Doch scheint mir die Deutung, dass diese Gestalt die Auferstehung darstellen soll, mehr für sich zu haben. Tod und Krankheit wären fast gleiche Begriffe und würden als Wiederholung erscheinen. Viel sinnvoller aber schmücken ein Grabdenkmal, das dem Tode dient, der Tod und neben ihm die Auferstehung, welche die den Schrecken des Todes und Grabes überwindende Hoffnung verkörpert. Die übrigen 4 Fullungen der Postamente sind mit je 2 Lilien und Tulpen geschmuckt, von denen die Abbilnüngen V. und VI. je ein Exemplar vorfuhren.

Zum Schluss noch einige Worte über die spăter hinzugefügten Gipsfiguren. Es sind Darstellungen Christian Albrechts und seiner Gemahlin von VilLERS. 1) Diese in völlig barocker Auffassung gehaltenen Arbeiten stören die strenge Schönheit des Denkmals und sind erst I67 I aufgestellt. Offenbar hat man gemeint, auf die breiten Gesimsvorsprünge Figuren stellen zu müssen. Die Rechnungsbücher berichten:

167129 Julii.

Auff des H. Praesidenten Anmelden und der quitierten Beylage, d'Vil.LERS fuir Zwo Fitrstl. Contrafaicte von Gips tiber das Portal fur die Erbbegräbnus in der Thumb Kirchen, Bedungenermaszen Zahlt. . . . 200 [Reichstaler].

Der untere Teil des schönen schmiedeeisernen Gitters (Abb. I.) ist ein Stück des

1) Wahrscheinlich ist gemeint der Architekt und Bildhauer Jean Aqnaud Villers aus Paris, Er słand in Diensten des Kurfürsten von Brandenburg und ging 1668 mit einem Empfehlüngsschreiben desselben nach Bayern. Vgl. die Lexika von Füssli und Nagler sowie Nicolal, Beschreibung der Kgl. Residenzstädte Berlin und Potsdam. Aabang, I786. 
Chorgitters. Dieses prächtig bemalte spätgotische Meisterwerk der Schmiedekunst stammt nach HAUPT aus dem Anfang des 16. Jahrhunderts. Der obere Teil des Gitters (Abb. II.) mit dem Schleswig-Holsteinischen Wappen wird in der gleichen Zeit wie das Portal entstanden sein.

Das Portal der Herzoglichen Gruft im Dom zu Schleswig, das wie der Bruggemannsche Altar und die Heilige Familie des Jürgen Ovens zu den schönsten Kunstwerken der Hauptkirche des Landes gehört, hat stets die Bewunderung der Kunstfreunde hervorgerufen. Sein hoher künstlerischer Wert ist seit Alters anerkannt worden. Erfreulich ist est daher, dass wir jetzt einige Klarheit uber die Entstehung des Portals gewonnen haben und den Namen des bedeutenden Künstlers kennen, Artus Quellinus.

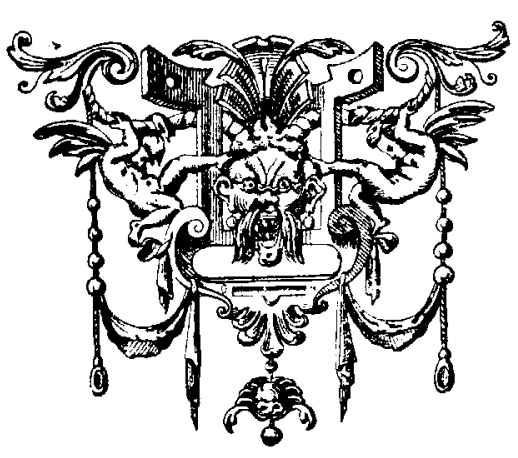

